УДК 538.9

\title{
Некоторые проблемы теоретической лазерной физики: от пичковой генерации к экстремальной и топологической лазерной оптике
}

\author{
(C) 2018 Г. Н. Н. РОЗАНОВ, ДОКТОР ФИЗ.-МАТ. НАУК, ЧЛЕН-КОРРЕСПОНДЕНТ РАН \\ АО «Государственный оптический институт им. С.И. Вавилова», Санкт-Петербург \\ Университет ИТМО, Санкт-Петербург \\ Физико-технический институт им. А.Ф. Иоффе Российской академии наук, Санкт-Петербург \\ E-mail: nnrosanov@mail.ru
}

Поступила в редакцию 08.07.2018

DOI:10.17586/1023-5086-2018-85-11-08-18

\begin{abstract}
Представлены краткий обзор теоретических исследований в Государственном оптическом институте и более подробное изложение недавних результатов в области теоретической лазерной физики и нелинейной оптики. Наиболее детально прослежено развитие теоретических представлений о бистабильности в пространственно распределенных оптических системах, вплоть до последних результатов по трехмерным топологическим лазерным солитонам и предельно коротким лазерным импульсам.
\end{abstract}

Ключевые слова: лазерная физика, экстремальная нелинейная оптика, оптическая бистабильность, диссипативные оптические солитоны, предельно короткие импульсы.

Коды OCIS: 140.0140, 190.0190, 260.0260, 320.0320

\section{ВВЕДЕНИЕ}

Как хорошо известно, и во время создания Государственного оптического института (ГОИ, 1918 г.), и в дальнейшем, в том числе в условно «лазерную» эпоху (включая голографию и нелинейную оптику, после 1960 г.), оптика находилась на переднем крае физики с высоким насыщением принципиальными и фундаментальными проблемами. Безусловно, это вызывало крайнюю необходимость серьезных теоретических исследований по широкому фронту связанных с оптикой проблем. В то же время отношение к теоретической деятельности в ГОИ было неоднозначным. Для его характеристики приведем мнение одного из старейших сотрудников ГОИ, впоследствии академика И.В. Обреимова [1], свидетельствующее о позиции идеолога и первого директора ГОИ, блестящего ученого и талантливого организатора науки Д.С. Рождественского:

«По отношению $к$ теоретической физике Дмитрий Сергеевич страдал общим недостатком русских физиков его поколения. Он относился к ней ... снисходительно ... А настоящих физиков-те- оретиков в то время в России не было. С этим связаны иллюзии Рождественского о том, что если бы он захотел, то мог бы работать в области теоретической физики. В ГОИ и до сих пор теоретическая физика не прижилась, хотя здесь работали В.А. Фок, затем в 30-40-х годах сильная группа физиков, занимавшаяся молекулярной спектроскопией ..., но в институте их не ценили и дали уйти».

Эта цитата относится к 1974 г., и подобная точка зрения разделялась рядом крупных ученых ГОИ: теоретики должны быть при экспериментаторах и обслуживать их потребности. Действительно, в экспериментальных подразделениях ГОИ были глубокие теоретики, как например В.П. Козлов (математическая статистика и обратные задачи оптики), имелись и счастливцы, одинаково сильные в теории и в эксперименте (Ю.Т. Мазуренко, широкий круг задач молекулярной спектроскопии и физической оптики; В.В. Любимов, теория открытых резонаторов для лазеров); исходно «чистый» теоретик, В.Г. Сидорович (теория обращения волнового фронта при нелинейном рассеянии света, при консультациях В.П. Козлова) затем 
ориентировался более на эксперимент, как и, в несколько иных обстоятельствах, Т.А. Вартанян (взаимодействие света с веществом) и др. Но масштаб задач, особенно в лазерный период, требовал полноценного участия профессиональных теоретиков. И здесь необходимо вспомнить их великих предшественников в ГОИ, к которым в первую очередь необходимо отнести члена-корреспондента АН CCCP В.С. Игнатовского и академика АН CССР В.А. Фока. Первый из них специализировался в области физической оптики (см. статьи в Трудах ГОИ [2-4], прокомментированные и углубленные В.А. Фоком [5]). Результаты B.C. Игнатовского впоследствии были «переоткрыты» и вошли в золотой фонд физики и оптики под другими именами; именно его можно причислить к пионерам сингулярной и топологической оптики, введшим в арсенал такие понятия (в современной нотации) как топологическая фаза и дислокации волнового фронта. Что же касается выполненных в ГОИ работ В.А. Фока, то они общеизвестны: так, найденные им сугубо квантовые «обменные силы» и мощный метод Хартри-Фока [6, 7] уже много лет служат «рабочей лошадью» при расчете спектров многочастичных квантовых систем. С дальнейшим развитием идей В.А. Фока в стенах ГОИ применительно к спектроскопии можно познакомиться по статьям [8, 9]. Мы не затрагиваем здесь большой объем работ по вычислительной оптике.

В период стабильного расширения исследований в ГОИ, в 1960-х гг., в ГОИ имелась теоретическая лаборатория, руководимая учеником В.А. Фока А.Г. Власовым. Наиболее известные работы А.Г. Власова относятся, скорее, к прикладным задачам: разработке теории и методов расчета электронной оптики и тонкослойных оптических покрытий. Преимущественно этими задачами занимались и ведущие сотрудники лаборатории Ю.А. Флегонтов и И.М. Минков. Сам же Анатолий Григорьевич особо дорожил своими исследованиями по математической физике, где им был предложен «метод переопределенных рядов». Пожалуй, «количественным» толчком к расширению лаборатории послужил прием в нее в 1963 г. большой группы выпускников кафедры теоретической физики В.А. Фока Ленинградского государственного университета. Это, вместе с поддержкой совместителей - учеников В.А. Фока, П.П. Павинского и А.В. Тулуба, и доброжелательным отношением к теории со стороны директора ГОИ М.М. Мирошникова, привело в начале 1970-х гг. к преобразованию лаборатории в теоретический отдел. Первоначально в составе отдела не предполагалось лаборатории лазерного направления; следует оговорить, что А.Г. Власову трудно было воспринять идеи этой новой области науки и он не разделял многие взгляды своих молодых сотрудников в этой области. Однако объективная необходимость в лазерной теории и авторитет Артура Афанасьевича Мака - многолетнего руководителя лазерного подразделения ГОИ - привели к созданию такой лаборатории, которая затем перешла в его отдел. Поскольку автор данной статьи лучше знаком с лазерной тематикой, то в дальнейшем я ограничусь изложением наиболее ярких «лазерных» результатов сотрудников этой лаборатории, не затрагивая теорию взаимодействия интенсивного лазерного излучения с веществом, чему посвящена отдельная статья в данном выпуске [10].

\section{ТЕОРИЯ ЛАЗЕРОВ}

Следует отметить, что А.А. Мак проявлял к теоретикам особое внимание и наставлял, что они должны быть не на обслуге экспериментаторов, а впереди них, генерируя новые идеи и предложения экспериментов. Тем не менее, первые работы молодых теоретиков в лазерной области были инициированы экспериментом. Не придерживаясь хронологии, отметим, прежде всего, блестящий результат В.А. Смирнова в поставленной задаче о возможности построения лазера на орто-пара переходах молекул водорода. Известна была оценка вероятности перехода, данная классиком квантовой механики Ю. Вигнером, которая делала задачу построения лазера хотя и трудной, но выполнимой. К работе были привлечены лучшие «лазерные» теоретики страны, включая А.Н. Ораевского и П.А. Апанасевича, которые на совещании согласились с оценкой Вигнера. И тут Валентин Анатольевич, тогда младший научный сотрудник, еще и не кандидат наук, заявил, что такая оценка некорректна из-за дополнительного запрета перехода, вызванного некоей скрытой симметрией, а правильный расчет показывает вероятность перехода на много порядков меньше. Как рассказывал В.А. Смирнов, сначала такое заявление вызвало смех, но через несколько дней, после проверки его выкладок, участники совещания подтвердили этот результат, закрывший проект указанного лазера.

Первой работой теоретиков лаборатории в области газовых лазеров было построение теории эффекта Зеемана для гелий-неонового лазера в магнитном поле. Работа проводилась по инициативе А.В. Тулуба, который дал ссылку на свежий эксперимент американских физиков и указал путь решения - обобщение недавно опубликованной теории газовых лазеров нобелевского лауреата У. Лэмба. Ввиду зеемановского расщепления атомных уровней уже в одномодовом режиме наблюдалась частота биений, которая, однако, странным образом зависела от напряженности магнитного поля. Анализ привел к неожиданному результату американцы не заметили изменения знака частоты биений, с учетом чего указанная зависимость 
существенно упрощалась. Наша статья [11] была опубликована в Докладах Академии наук (ДАН) СССР по представлению акад. А.А. Лебедева (ГОИ); одновременно в Журнале экспериментальной и теоретической физики опубликована независимая статья теоретиков ФТИ им. А.Ф. Иоффе М.И. Дьяконова и В.И. Переля, содержащая аналогичный вывод. Еще одна наша статья с уточнением теории за счет более высоких порядков теории возмущений была опубликована также в ДАН СССР по представлению В.А. Фока [12].

Статья [11] послужила завязкой контактов с ведущими специалистами ГОИ в области газовых лазеров - И.М. Белоусовой и О.Б. Даниловым. Так, еще одной работой в области газовых лазеров была развитая по инициативе О.Б. Данилова и И.М. Белоусовой теория взаимодействия поперечных мод газового лазера [13], также являвшаяся обобщением теории У. Лэмба. Кроме того, О.Б. Данилов привлек нас к решению поставленной перед А.А. Лебедевым проблемы, связанной с применением кольцевых лазеров в качестве датчика угловой скорости - гироскопа. Хотя расщепление частоты встречных волн идеального вращающегося резонатора пропорционально измеряемой угловой скорости, лазерный гироскоп «не работал» в области малых расщеплений из-за эффекта захвата частот, вызванного паразитным встречным рассеянием излучения и нелинейными эффектами. Наш анализ показал, что в области захвата возможно определение угловой скорости по разности фаз встречных волн (с совпадающими частотами) [14]. При обсуждении на семинаре А.А. Лебедев попросил пояснить эффект без формул, по аналогии с камертонами. Докладчик (автор настоящей статьи) с такой задачей не справился, хотя сейчас, видимо, и смог бы дать разъяснения со ссылкой на давний эксперимент Гюйгенса с двумя маятниками, подвешенными на единую опору.

В то же время в лазерном отделе ГОИ проводились интенсивные исследования динамики излучения твердотельных лазеров. Центральной здесь была проблема пичковой генерации в непрерывном режиме, причина которой была не ясна. Эта задача была поставлена А.А. Маком и перед теоретиками лаборатории, а решить ее удалось Г.Н. Винокурову с соавторами [15]. Г.Н. Винокуров на основе компьютерных расчетов показал чрезвычайную чувствительность динамики твердотельного лазера, обладающего слабо затухающими релаксационными колебаниями, к нестабильности параметров лазера, что и было признано причиной пичковой генерации. Этот фундаментальный результат открыл дорогу к получению стабильной (безпичковой) генерации и последующей серии блестящих экспериментов ГОИ по сверхчувствительной внутрирезонаторной лазерной спектроскопии. Затем несколько иной подход к пробле- ме пичковости был предложен автором данной статьи. В этом подходе шумы отсутствовали, а неустойчивость стационарного режима вызывалась наличием запаздывающего отклика от зеркал, дополнительных к основным зеркалам резонатора. В общем случае была развита теория динамики лазера с частотной дисперсией резонатора [16], которая в упрощенном варианте лазера с дополнительным зеркалом описывалась уравнением с запаздыванием [17]. Заметим, что аналогичное уравнение, сформулированное применительно к полупроводниковым лазерам, было опубликовано за рубежом заметно позже и часто цитируется как уравнение Ланга-Кабаяши (см. обзор в работе [18]).

Еще одной актуальной для лазерных работ ГОИ задачей была проблема мелкомасштабной фокусировки излучения мощных твердотельных лазеров, из-за которой исходно гладкий лазерный пучок разваливался на отдельные нити, или филаменты. Исходной здесь служила заметка В.И. Беспалова и В.И. Таланова (Институт прикладной физики АН) [19]. В этой области В.А. Смирнов и автор данной статьи развили теорию мелкомасштабной фокусировки лазерных пучков (в отличие от плоской волны) [20] и последовательную теорию мелкомасштабной самофокусировки в многоэлементных нелинейных системах [21], которая затем использовалась как в ГОИ, так и в других институтах; ряд результатов в этой области получил также в ГОИ Л.А. Нестеров. Примыкает к этой деятельности предложенная нами теория мелкомасштабной неустойчивости нелинейных поверхностных волн [22] и различные аспекты теории консервативных оптических и родственных солитонов (в том числе солитонов конденсата Бозе-Эйнштейна в разреженных ультрахолодных газах [23-27]). Была показана реальность ряда нелинейно-оптических явлений даже «без среды» - в электрон-позитронном вакууме [28-30]. Определенный цикл образуют проведенные исследования релятивистских эффектов в оптике, которые можно разделить на несколько разделов. Во-первых, это оптика сред с пространственной неоднородностью скорости движения, включая геометрическую оптику таких сред (см. обзор [31]). В частности, интерес представляет уточнение [32] описания классического опыта Физо, продемонстрировавшего частичное увлечение света движущейся средой. В этом эксперименте свет распространяется вдоль трубы с движущейся жидкостью, из-за чего скорость распространения света вдоль и против движения жидкости оказывается различной. Уточнение состоит в том, что скорость ламинарного течения жидкости зависит от расстояния от оси, принимая максимальное значение на оси и убывая к стенкам трубы. Поэтому текущая жидкость создает для света эффективную распределенную линзу, фокусное расстояние которой различно в противоположных 
направлениях распространения света (невзаимный элемент). Подобные релятивистские эффекты могут оказаться полезными для диагностики неоднородности скорости движения жидкостей и имеют аналоги для акустических волн в гидродинамике [31]. Второй раздел относится к теории отражения и рассеяния света на движущихся с релятивистской скоростью неоднородностях среды, которые могут быть наведены в нелинейной среде интенсивными лазерными импульсами [33-38]. Наконец, третий раздел посвящен теории классического аналога квантового динамического эффекта Казимира [39], согласно которому в пустом резонаторе с движущимся зеркалом может рождаться излучение за счет преобразования кинетической энергии зеркала в электромагнитную энергию [40, 41]. В работах «на злобу дня» $[42,43]$ была показана принципиальная невозможность идеальной невидимости даже в варианте монохроматического излучения.

Лаборатория активно участвовала и в комплексных темах лазерного и других отделов ГОИ, обеспечивая масштабное численное моделирование различных систем; здесь нужно особо отметить вклад талантливого физика и компьютерного виртуоза В.Е. Семенова. Коллектив имел тесные контакты с ведущими теоретиками Физического института АН, Московского государственного университета, Института прикладной физики $\mathrm{AH}$ и др. и входил, по нашему мнению, в число ведущих в стране в области динамики лазерных и нелинейно-оптических систем, а также по теории и методам расчета нелинейного распространения лазерного излучения.

\section{ОТ БИСТАБИЛЬНОСТИ К ЭКСТРЕМАЛЬНОЙ И ТОПОЛОГИЧЕСКОЙ НЕЛИНЕЙНОЙ ОПТИКЕ}

Основные крупные циклы «собственных» теоретических работ, пожалуй, начались с теории отражения плоской волны от нелинейной среды. И.М. Минков на семинаре лаборатории рассказал о появившейся статье на эту тему. Эта задача включала ряд нетривиальных моментов и требовала серьезного, последовательного анализа. Доложенные на семинаре результаты вызвали у автора данной статьи сомнения. Родственность уравнений анализировавшимся ранее [16] позволила установить, что, действительно, из четырех сценариев отражения, возможных при различных комбинациях параметров, два были описаны ранее некорректно. Последовательный анализ, в котором привлекался известный в математике «принцип предельного поглощения», был впервые опубликован в наших статьях $[44,45]$ и затем вошел в монографии $[46,47]$.

Решение в приближении плоских волн показало возможность бистабильности и мультистабильности - установления одного из нескольких режимов отражения в зависимости от начальных условий (устойчивость режимов мы здесь не обсуждаем). И это породило новые вопросы. Главный из них - как происходит гистерезис не в точечной, а в пространственно распределенной системе? Действительно, для оптики характерны пучки с шириной, значительно превосходящей длину волны. Такой пучок можно разбить на отдельные лучевые трубки, в каждой из которых в случае би- или мультистабильности, казалось бы, может устанавливаться тот или иной режим. Но тогда не ясно, где же проходит пространственная граница между режимами? Этот вопрос пыталась решить одновременно с нами другая группа, пришедшая к парадоксальному выводу: в случае падения на нелинейную среду пучка (в отличие от плоской волны) би- и мультистабильность исчезают - режим становится единственным. Однако в нашей работе [48] вывод был иным: бистабильность возможна и для пучка с ограниченной шириной, но анализ требует учета более тонких эффектов типа пограничного слоя (другими словами, наши оппоненты заранее пренебрегли возможностью резких гистерезисных скачков поля, не описываемых в принятом ими приближении медленно меняющейся огибающей). Вместе с дальнейшим развитием этих идей, в котором приняли участие мои коллеги В.Е. Семенов, Г.В. Ходова, А.В. Федоров, С.В. Федоров, А.Н. Шацев, А.Г. Владимиров, Л.А. Нестеров, Н.А. Веретенов и др., удалось построить достаточно общую теорию пространственного гистерезиса и бистабильности пространственно распределенных систем, применимость которой, на наш взгляд, не ограничивается оптическими схемами. Теория развивалась в нескольких направлениях, ряд которых мы рассмотрим ниже (см. также монографии [49, 50]).

Простейшей схемой, в которой нами был (теоретически) продемонстрирован пространственный гистерезис, был тонкий полупроводниковый стержень или пластина с коэффициентом поглощения, растущим с температурой, с нагревом через боковую поверхность широким пучком излучения $[51,52]$. При малой длине стержня или же при однородном нагреве может иметь место бистабильность: исходно холодный полупроводник почти не поглощает излучения и остается холодным, тогда как исходно нагретый полупроводник поглощает значительную часть излучения и поддерживает горячее состояние при том же уровне облучения. Но при однородном нагреве стрежня большой длины можно обеспечить исходно холодное состояние одной половины стержня и горячее состояние его другой половины. Тогда в стержне формируется волна переключения: фронт между холодной и горячей областями стержня движется вдоль оси стержня со скоростью, которая зависит от интенсивности облучения знакопеременным образом. 
Это, по сути, решает проблему пространственного гистерезиса при нагреве длинного стержня широким пучком, локально приближаемым плоской волной с соответствующей интенсивностью. А именно, переключение между режимами происходит там, где локальная интенсивность равна «максвелловскому» значению, при котором скорость волны переключения обращается в ноль. Если накачка плавно промодулирована по поперечному направлению, то возможны локализованные структуры - островки одного из режимов, пространственно сосуществующие с другими режимами. Эти структуры можно считать диссипативными солитонами, поскольку механизмом локализации служит баланс между линейным (диффузионным) расширением островка и его нелинейной фокусировкой за счет уравновешивания притока и оттока энергии в области локализации. Заметим, что понятия волн переключения и пространственного гистерезиса оказываются полезными в различных отраслях науки, включая нанотехнологии [53].

Решение этой задачи вызвало новые, притом более трудные вопросы применительно к родственным системам. В рассмотренном примере внешнее излучение может быть некогерентным. Нетривиальные особенности возникают в чисто оптических схемах с когерентным излучением, в которых поперечные эффекты определяются уже не диффузией, а дифракцией. Сначала наша группа впервые продемонстрировала диссипативные солитоны в широкоапертурном интерферометре, заполненном нелинейно-оптической средой и возбуждаемом внешним когерентным излучением [54]. Как и в предыдущей схеме, такие солитоны во многих случаях полезно интерпретировать как связанные волны переключения [55]. Но теперь эти волны обладают дифракционными осцилляциями, которые и «сцепляют» волны переключения. Исторически сначала Г.В. Ходова изучала столкновение волн переключения между стационарными и нестационарными состояниями и получила сосуществующие в пространстве островки стационарных, периодических и хаотических режимов [56, 57], в последнее время получившие название «химер». Затем родственные солитоны были найдены нами в широкоапертурном лазере с насыщающимся поглощением [58]. Такие диссипативные солитоны оказываются «калиброванными» - их, например, размеры принимают ряд дискретных значений, что ведет к повышенной устойчивости этих структур. Поскольку размеры диссипативных оптических солитонов в полупроводниковых микрорезонаторах порядка 10 мкм и они могут быть еще уменьшены, то здесь открываются возможности использования таких солитонов для разнообразных информационных приложений. Эта тема получила далее широкое развитие в различных направлениях, результаты суммированы по состоянию на 2011 г. в монографии [49]. Повышенная устойчивость диссипативных солитонов подтверждается и их большей устойчивостью к квантовым флуктуациям поля. Рассматриваемые солитоны формируют комплексы, которые могут быть «жесткими» - не меняющими форму при эволюции, вызывающей только их движение и вращение (в поперечно двумерной геометрии). Механика таких комплексов определяется симметрией распределений интенсивности и потока энергии поля (вектора Пойнтинга). В указанной геометрии с двумя элементами симметрии (ось симметрии и симметрия к повороту на угол, дробный от $2 \pi$ ) возникает 4 варианта поперечной динамики: неподвижность, равномерное прямолинейное движение с постоянной скоростью, вращение с постоянной угловой скоростью вокруг неподвижного центра и «лунное движение» - движение центра структуры по окружности с одновременным вращением с тем же периодом. Анализ топологии потоков энергии позволяет также строго разделить случаи слабого и сильного взаимодействия диссипативных (прежде всего, лазерных) солитонов.

И эти результаты привели к постановке принципиальных и еще более сложных вопросов. В последнее время внимание физиков все более занимают топологические проблемы, а недавно зародилось перспективное направление топологической фотоники. Примыкает к нему и тематика трехмерных топологических лазерных солитонов. Однако, если традиционно топологическая нетривиальность достигается за счет наноструктурирования оптической среды [59], то в случае солитонов речь идет о сложной топологии самого поля, реализующейся даже в однородной среде. C «академической» точки зрения такие солитоны интересны как модель весьма разнообразных самоорганизующихся структур со сложной внутренней организацией. Важный класс узловых солитонов был введен Л.Д. Фаддеевым [60] при попытке раскрытия структуры элементарных частиц. Для приложений важно, что, во-первых, такие структуры обладают, дополнительно к особой устойчивости диссипативных солитонов, свойством сохранения топологических характеристик даже при не малых возмущениях. Во-вторых, их разнообразие допускает использование их в качестве символов или «иероглифов» для компактной записи и обработки информации.

Ниже представлены последние результаты нашей группы в этой области [61-64]. Солитоны формируются в однородной среде с насыщающимися усилением и поглощением или же в лазере с достаточно длинным резонатором (время обхода света по резонатору превышает времена релаксации среды). Основным уравнением, описывающим пространственно-временную динамику структур света, является обобщенное комплексное уравне- 
ние Гинзбурга-Ландау для медленно меняющейся огибающей напряженности электрического поля $E$ (считаем излучение близким к линейно поляризованному)

$$
\frac{\partial E}{\partial z}=\left[\left(i+d_{\perp}\right) \Delta_{\perp}+\left(i+d_{\|}\right) \frac{\partial^{2}}{\partial \tau^{2}}\right] E+f E .
$$

Здесь все величины представлены в безразмерной форме. Основное направление распространения излучения - вдоль оси $z, \Delta_{\perp}=\partial^{2} / x^{2}+\partial^{2} / \partial y^{2}-$ поперечный лапласиан, $x$ и $y-$ поперечные координаты, $\tau=t-z / v_{g r}$ - время в системе координат, бегущей вдоль оси $z$ с групповой скоростью $v_{g r}$, $t$ - время в лабораторной системе координат. «Эффективные коэффициенты диффузии» $d_{\perp}, d_{\|}$ отвечают дисперсии показателя преломления среды $\left(d_{\|}\right)$и дихроизму $\left(d_{\perp}\right)-$ угловой селективности потерь излучения, $0<d_{\perp}, d_{\|}<<1$. В случае быстрого отклика среды (лазеры класса А) можно считать $f$ мгновенной функцией интенсивности излучения $I=|E|^{2}$, т.е. $f=f\left(|E|^{2}\right)$. При точной частотной настройке эта функция вещественная

$$
f\left(|E|^{2}\right)=-1-\frac{a_{0}}{1+b|E|^{2}}+\frac{g_{0}}{1+|E|^{2}} .
$$

В правой части формулы (2) член (-1) представляет нерезонансное поглощение, $a_{0}$ - линейный коэф-

(a)

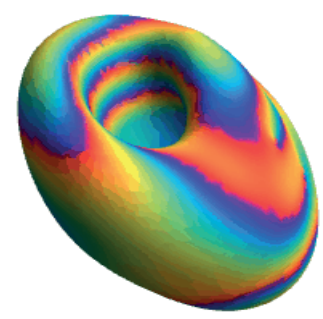

(д)

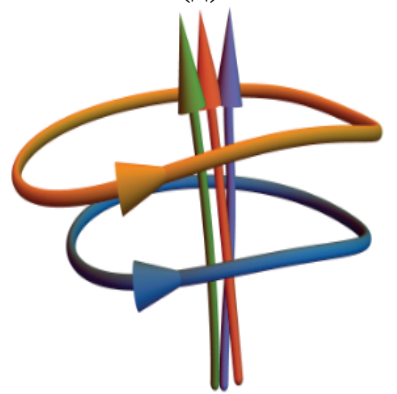

(б)

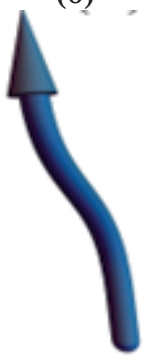

(e)

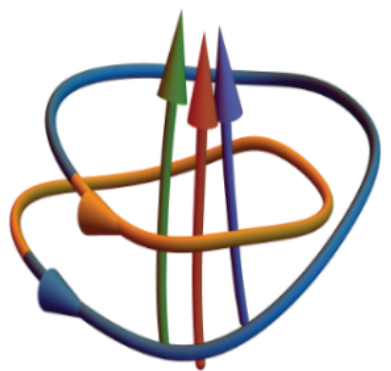

фициент насыщающегося поглощения, $g_{0}-$ линейный коэффициент насыщающегося усиления, $b$ - отношение интенсивностей насыщения усиления и поглощения. Выбираем такие условия, когда при слабой интенсивности потери превышают усиление

$$
1+a_{0}>g_{0}
$$

что обеспечивает устойчивость безгенерационного режима $E=0$. Но с ростом интенсивности потери убывают быстрее, чем усиление, поэтому может реализоваться режим жесткого возбуждения генерации. Лазерный солитон можно качественно представить как стабильный островок такой генерации на фоне безгенерационного режима.

Наши поиски основывались на построении указанных солитонов с помощью изученных ранее двумерных лазерных солитонов [49]. Сложность топологии трехмерных структур заставляет искать их более простые сингулярные элементы. В качестве последних удобно выделение вихревых линий, на которых поле обращается в нуль, и отдельных сингулярных точек на них, в окрестности которых меняется знак касательной компоненты потока энергии. Вихревые линии ориентированы в направлении возрастания фазы поля.

Внешняя форма солитонов представлена только на двух рисунках (рисунки а, в), поскольку формы различных типов солитонов различаются

(B)

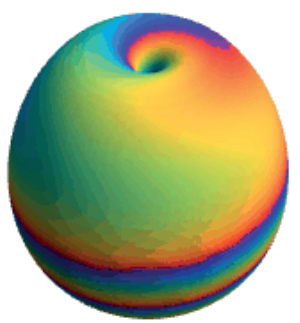

(ж)

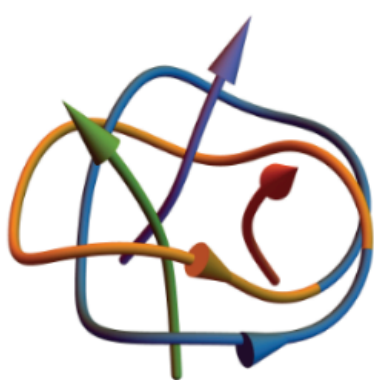

(г)

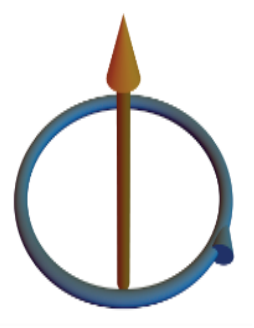

(3)

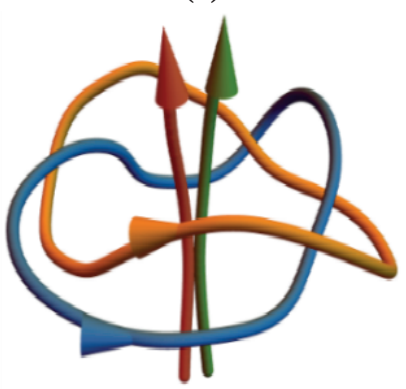

Поверхности изоинтенсивности, показывающие внешние размеры солитонов (а, в) и их «скелеты» - наборы вихревых линий в проекции на плоскость $\left(б, \Gamma_{-3}\right)$. Рисунки $(a$, б) иллюстрируют «прецессон» с единственной незамкнутой вихревой линией, $($ в, г) - «яблоко» с одной незамкнутой и одной замкнутой вихревыми линиями, (д) - солитон с двумя незацепленными замкнутыми и тремя незамкнутыми вихревыми линиями, (е) - солитон с тремя незамкнутыми и одной замкнутой линией с топологией тривиального узла, $(ж)-$ солитон с тремя незамкнутыми и одной замкнутой узловой (трилистник) вихревыми линиями, (з) - «соломонов солитон» с двумя незамкнутыми и двумя замкнутыми зацепленными линиями. Цвет на рисунках (а, в) отражает фазу поля, а стрелки на вихревых линиях их ориентацию с возрастанием фазы вблизи вихревых линий. 
слабо. В то же время разнообразие «скелетов» солитонов, формируемых совокупностью вихревых линий, позволяет характеризовать их хотя и не исчерпывающе, но с достаточной для классификации полнотой. Общим свойством приведенных на рисунке основных типов топологических солитонов служит наличие вихревых линий. При обходе по замкнутому контуру вокруг этих линий фаза поля меняется на $2 \pi$, что отвечает вихрю потока энергии. Представленные вихревые линии разделяются на замкнутые и незамкнутые (уходящие на периферию солитона), причем незамкнутые линии проходят внутри замкнутых.

Простейшим из иллюстрированных рисунком солитонов является «прецессон» (рисунки а, б). $\mathrm{У}$ него распределение интенсивности асимметрично и вращается в некоторой области параметров с прецессией и имеется единственная искривленная вихревая линия (незамкнутая). На рисунках $(в$, г) представлен «солитон-яблоко» с одной незамкнутой и одной замкнутой вихревыми линиями. При определенных параметрах незамкнутая линия является прямой, замкнутая - окружностью, и структура поля обладает осевой симметрией. Изменение параметров схемы ведет к топологически эквивалентным структурам с деформированной преимущественно замкнутой или же незамкнутой линиями. «Скелет» солитона с двумя незацепленными замкнутыми (их можно развести на произвольно большое расстояние без разрывов и самопересечений) и тремя незамкнутыми вихревыми линиями представлен на рисунке (д). Более точно - это нестационарная структура, в ней замкнутые линии, периодически сжимаясь и расширяясь, проходят друг сквозь друга. На рисунке (е) изображен солитон с тремя незамкнутыми и одной замкнутой вихревыми линиями. В отсутствие незамкнутых линий деформацией без разрывов и пересечений замкнутую линию можно превратить в круг, так что она обладает топологией тривиального узла. На рисунке (ж) число замкнутых и незамкнутых вихревых линий такое же, как для (е). Однако теперь замкнутую линию нельзя плавными деформациями преобразовать в круг, так что это узел (трилистник). Наконец, на рисунке (з) продемонстрирован «соломонов солитон» с двумя незамкнутыми и двумя замкнутыми зацепленными (их нельзя развести на большое расстояние без разрывов) линиями. Скелеты этих структур, кроме «прецессона», можно отнести к тэнглам («запутанные клубки») - так в топологии именуют наборы замкнутых и незамкнутых линий. Более детальный анализ показывает и наличие на вихревых линиях отдельных сингулярных точек, в окрестности которых меняется знак касательного к линии потока энергии.

Представленные типы солитонов существуют и устойчивы в определенных областях параметров системы, причем эти области перекрываются. Естественно ожидать, что при плавном изменении параметров одни структуры преобразуются в другие. При этом должны наблюдаться бифуркации структур и гистерезисные явления. Наши последние расчеты подтверждают эти ожидания, выявляя при этом новые интересные особенности перестройки топологии структур. При изменении контрольного параметра (линейного коэффициента усиления) происходят бифуркации вихревых линий нескольких типов. Первый тип - их антипараллельное переключение, когда две приближающиеся друг к другу линии с противоположной направленностью вблизи точки касания обмениваются ветвями. Во втором типе вихревая линия изгибается так, что формируется отрывающаяся от нее петля. В третьем - изменение топологии может вызываться приходом сингулярностей с далекой периферии солитона. В результате серии таких бифуркаций при медленном возрастании линейного коэффициента усиления (накачки) исходный «соломонов солитон» превращается в два разлетающихся «солитона-яблока». При последующем убывании коэффициента усиления эти два солитона с более простой топологией сохраняются вплоть до границ своего существования, так что гистерезисная петля не образуется.

Характеризуя состояние этого направления, следует отметить, что оно находится только в начале пути: многие важные аспекты (например, конечность времен релаксации среды) еще не изучены теоретически, а экспериментальные исследования этого необычного класса трехмерных топологических солитонов вообще отсутствуют.

Еще одно ответвление темы диссипативных оптических солитонов связано с их экстремальными состояниями. Здесь можно отметить, во-первых, поиск и анализ солитонов с предельно малыми размерами вплоть до молекулярных. Подобные диссипативные «наносолитоны» были теоретически продемонстрированы в работах $[65,66]$ применительно к молекулярным Ј-агрегатам, а подробный анализ более тонких механизмов многочастичного межмолекулярного взаимодействия в них выполнен в работах $[67,68]$.

Особое место занимает в этом ряду чрезвычайно актуальная и сложная тема предельно коротких лазерных импульсов и диссипативных солитонов, которые могут иметь наибольшие значения пиковой интенсивности. В этой области нарушаются многие традиционные для оптики приближения (однонаправленного распространения, медленно меняющейся огибающей и др.), что требует пересмотра сложившихся представлений. В теории во многих случаях необходимо обращаться к строгим уравнениям Максвелла электродинамики сплошных сред. Интересно, что их общий вид допускает вывод некоторых общих 
правил сохранения для полей, локализованных в конечной пространственной и временно́й области. Сама возможность законов сохранения в системах с диссипацией, видимо, контринтуитивна ввиду, например, затухания поля в протяженной среде с поглощением. Однако интегрирование по объему уравнения Максвелла

$$
\operatorname{rot} \mathbf{E}=-\frac{1}{c} \frac{\partial \mathbf{B}}{\partial t}
$$

приводит к выводу (E и $\mathbf{B}-$ напряженность электрического и индукция магнитного полей) о сохранении «объема магнитного поля»

$$
\mathbf{V}_{B}=\iiint_{V} \mathbf{B} d \mathbf{r}, \frac{d}{d t} \mathbf{V}_{B}=\mathbf{0} .
$$

Интегрирование же уравнения (4) по времени ведет к соотношению

$$
\operatorname{rots}_{E}=0 \text {, }
$$

где введена «электрическая площадь» импульса

$$
\mathbf{S}_{E}=\int_{t=-\infty}^{+\infty} \mathbf{E} d t
$$

Для популярного в теоретическом анализе приближения одномерной геометрии (поля зависят только от одной продольной координаты $z$ ) формула (7) принимает вид правила сохранения

$$
\frac{d}{d z} \mathbf{S}_{H}=0
$$

Эти и родственные правила сохранения выведены и проанализированы в работах [69-71, 49]. В условиях применимости однонаправленного приближения из сохранения электрической площади следует, например, что в поглощающей среде уменьшение амплитуды поля по мере распространения должно сопровождаться таким же удлинением импульса и убыванием энергии импульса. Отметим, что «электрическая площадь» существенно определяет и эффективность возбуждения квантовых объектов короткими импульсами [72].

Предельно короткие, вплоть до униполярных, диссипативные оптические солитоны («аттосолитоны») удалось получить теоретически в условиях, когда на известный эффект самоиндуцированной прозрачности накладываются диссипативные факторы - усиление и поглощение излучения в среде [73, 74, 49]. В указанных работах такие импульсы получены в «однопроходовых» схемах, хотя впоследствии предельно короткие импульсы удалось получить и для лазеров с «когерентным» насыщающимся поглощением [75]. Эти результаты открывают новые возможности не только для генерации предельно коротких лазерных импульсов, но и для формирования униполярных импульсов, действие которых на заряды однонаправленно (в отличие от случая стандартных биполярных импульсов). Униполярные импульсы могут быть получены разделением биполярных, состоящих из нескольких «полупериодов» с противоположными знаками напряженности основной поляризационной компоненты поля, на отдельные «полупериоды», а также рядом других методов [76]. Тем не менее, разработка эффективных подходов к генерации и усилению таких импульсов (включая упомянутый выше классический аналог эффекта Казимира) остается в числе интригующих задач этого раздела.

\section{ЗАКЛЮЧЕНИЕ}

Представляется, что приведенные в статье результаты теоретической деятельности в ГОИ им. С.И. Вавилова последних лет позволяют иначе оценить их уровень по сравнению с выводом, сделанным в свое время И.В. Обреимовым [1]. По нашему мнению, этот уровень отвечает современному, и в ряде важных направлений теория здесь идет впереди эксперимента. В конкретных условиях страны и ГОИ это отчасти объясняется тем, что для теоретических исследований требуется заметно меньшее финансирование, чем для экспериментов. Несмотря на значительные организационно-финансовые трудности, описанные выше теоретические исследования развивались в ГОИ с последовательным нарастанием их глубины, что вызвано, в первую очередь, внутренней логикой науки. В то же время необходимо подчеркнуть, что теоретический анализ и компьютерное моделирование позволяют ускорить и удешевить проектирование лазерных и оптических схем и предсказать новые направления развития современной оптики. Новые вызовы и возможности, связанные с экстремальной и топологической лазерной и нелинейной оптикой, позволяют надеяться на дальнейшее успешное развитие этого направления.

Автор благодарен всем коллегам и особенно С.В. Федорову за многолетнюю совместную работу. Исследования лазерных топологических солитонов поддержаны грантом РНФ 18-12-00075.

\section{ЛИТЕРАТУРА}

1. Обреилов И.В. Дмитрий Сергеевич Рождественский // Труды ГОИ им. С.И. Вавилова. 1974. Т. 42. № 175. С. 3-30.

2. Игнатовский В.С. Связь между геометрической и волновой оптикой и дифракция гомоцентрического пучка // Труды ГОИ. 1920. Т. 1. № 3. С. 1-30.

3. Игнатовский В.С. Дифракция объектива при любом отверстии // Труды ГОИ. 1919. Т. 1. № 4. С. 1-36. 
4. Игнатовский В.С. Дифракция параболического зеркала при любом отверстии // Труды ГОИ. 1920. Т. 1. № 5. С. 1-30.

5. Фок В.А. Исследование В.С. Игнатовского. Связь между геометрической и волновой оптикой и дифракция гомоцентрического пучка. (Новое изложение). Исследование В.С. Игнатовского. Дифракция объектива при любом отверстии. (Новое изложение) // Труды ГОИ. 1924. Т. 3. № 27. С. 1-51.

6. Фок В.А. Приближенный способ решения квантовой задачи многих тел // Труды ГОИ. 1931. Т. 5. № 51. С. 1-28.

7. Фок В.А. Применение обобщенного способа Хартри к атому натрия // Труды ГОИ. 1931. Т. 5. № 51. С. $29-39$.

8. Груздев П.Ф., Шерстюк А.И. Влияние идей В.А. Фока на развитие работ по теоретической атомной спектроскопии в Государственном оптическом институте // Труды ГОИ. 1978. Т. 43. № 177. С. 80-87.

9. Старостин Н.В., Онопко Д.Е. Влияние идей В.А. Фока на развитие работ по теоретической спектроскопии конденсированных сред // Труды ГОИ. 1978. Т. 43. № 177. С. 88-93.

10. Андреев А.А. Сверхсильный свет (достижения и перспективы) // Оптический журнал. 2018. Т. 85. № 11. С. Этот выпуск (к 100-летию ГОИ).

11. Розанов Н.Н., Тулуб А.В. К теории эффекта Зеемана в газовых лазерах // ДАН СССР. 1965. Т. 165. № 6. С. 12801283.

12. Розанов Н.Н., Тулуб А.В. О теории возмущений для поляризации в резонансных средах // ДАН СССР. 1968. Т. 181. № 4. C. $830-832$.

13. Белоусова И.М., Винокуров Г.Н., Данилов О.Б., Розанов Н.Н. Взаимодействие типов колебаний в газовом ОКГ с резонатором со сферическими зеркалами // ЖЮЭТФ. 1967. Т. 52. № 5. С. 1146-1156.

14. Розанов Н.Н., Винокуров Г.Н., Данилов О.Б. О фазовых соотношениях в зоне захвата кольцевого лазера // Опт. спектр. 1967. Т. 23. № 4. С. 624-626.

15. Винокуров Г.Н., Галактионова Н.М., Егорова В.Ф., Мак А.А., Седов Б.М., Ханин Я.И. Пичковая структура излучения твердотельного лазера // ЖЭТФ. 1971. Т. 60. № 2. С. 489-499.

16. Розанов Н.Н. О пульсациях излучения лазера с частотной дисперсией // ЖЭТФ. 1972. Т. 63. № 6. С. $2033-2042$.

17. Розанов Н.Н. О кинетике твердотельного лазера с дополнительным движущимся зеркалом // Квант. электрон. 1974. T. 1. № 10. C. 2143-2147.

18. Erneux T., Javaloyes J., Wolfrum M., Yanchuk S. Introduction to focus issue: Time-delay dynamics // Chaos. 2017. V. 27. P. 114201.

19. Беспалов В.И., Таланов В.И. О нитевидной структуре пучков света в нелинейных жидкостях // Письма в ЗЖЭТФ. 1966. T. 3. № 12. С. 471-476.

20. Розанов Н.Н., Слирнов В.А. Распад ограниченных лазерных пучков в нелинейной среде // ЖЮЭТФ. 1976. Т. 70. № 6. C. 2060-2073.

21. Розанов Н.Н., Слирнов В.А. Мелкомасштабная самофокусировка лазерного излучения в усилительных системaх // Квант. электрон. 1980. Т. 7. № 2. С. 410-419.

22.Высотина Н.В., Розанов Н.Н., Слирнов В.А. О неустойчивости распространения нелинейных поверхностных волн // Письма в ЖТТ. 1984. Т. 10. № 19. С. 1206-1209.

23. Rosanov N.N. Vector solitons of a Bose-Einstein condensate in a dynamical trap // Phys. Rev. A. 2014. V. 89. P. 035601.

24. Розанов Н.Н., Высотина Н.В. Солитон в стационарной и динамической ловушках // Письма в ЗЖЭТФ. 2014. Т. 100. № 8. C. 566-569.

25. Rosanov N.N., Vysotina N.V. Fermi-Ulam problem for solitons // Phys. Rev. A. 2015. V. 91. № 1. P. 013622.

26. Rosanov N.N., Vysotina N.V. Recurrence for motion of solitons of the Bose-Einstein condensate in a dynamic trap // JOSA B. 2015. V. 32. № 5. P. B20-B24.

27. Розанов Н.Н., Высотина Н.В. Левитирующий солитон конденсата Бозе-Эйнштейна // ЖЭТФ. 2016. Т. 150. № 1. C. 60-68.

28. Розанов Н.Н. Четырехволновые взаимодействия излучения в вакууме // ЖЭТФ. 1993. Т. 103. № 6. С. $1996-2007$.

29. Розанов Н.Н. О самовоздействии интенсивного электромагнитного излучения в электрон-позитронном вакууме // ЖЭЭТФ. 1998. Т. 113. № 2. С. 513-520.

30. Koga J.K., Bulanov S.V., Esirkepov T.Zh., Pirozhkov A.S., Kando M., Rosanov N.N. Possibility of measuring photonphoton scattering via relativistic mirrors // Phys. Rev. A. 2012. V. 86. № 5. P. 053823.

31. Розанов Н.Н., Сочилин Г.Б. Релятивистские эффекты первого порядка в электродинамике сред с неоднородной скоростью движения // УФН. 2006. Т. 176. № 4. С. 421-439.

32. Розанов Н.Н., Сочилин Г.Б., Данилов О.Б. Невзаимные волноводы и линзы на основе эффекта Френеля-Физо // Опт. спектр. 2003. Т. 95. № 6. С. 908-910. 
33. Розанов Н.Н. Преобразование электромагнитного излучения на движущихся неоднородностях среды // Письма в ЗЖЭТФ. 2008. Т. 88. № 8. С. 577-580.

34. Розанов H.Н. Преобразование электромагнитного излучения на быстро движущихся неоднородностях прозрачной среды // ЖЖЭТФ. 2009. Т. 135. № 1. С. 154-163.

35. Высотина Н.В., Розанов Н.Н. Попутное отражение света от движущейся неоднородности // Письма в ЖҢЭТФ. 2011. T. 93. № 6. C. 341-345.

36. Розанов Н.Н. Сверхсветовой параметрический эффект Доплера в диэлектриках и в электрон-позитронном вакууме // Письма в ЖЮЭТФ. 2012. Т. 95. № 12. С. 689-692.

37. Розанов Н.Н. Досветовой и сверхсветовой параметрические эффекты Доплера при отражении света от движущейся плавной неоднородности среды // ЖЭЭТФ. 2012. Т. 142. № 6(12). С. 1101-1107.

38. Буланов С.В., Есиркепов Т.Ж., Кандо М., Пирожков А.С., Розанов Н.Н. Релятивистские зеркала в плазме - новые результаты и перспективы // УФН. 2013. Т. 183. № 5. С. 449-486.

39. Красильников В.Н. Параметрические волновые явления в классической электродинамике. СПб.: Издательство С.-Петербургского университета, 1996. 300 с.

40. Розанов Н.Н., Мацковский А.А., Малевич В.Л., Синицын Г.В. О параметрическом возбуждении поля в резонаторе с осциллирующими зеркалами // Опт. спектр. 2015. Т. 119. № 1. С. 93-95.

41. Розанов Н.Н., Федоров Э.Г., Мацковский А.А. Параметрическая генерация излучения в динамическом резонаторе с частотной дисперсией // Квант. электрон. 2016. Т. 46. № 1. С. 13-15.

42. Розанов Н.Н. Невидимость: за и против // Природа. 2008. № 6. С. 3-10.

43. Розанов Н.Н. Достижима ли идеальная невидимость даже при монохроматическом излучении // УФН. 2011. T. 181. № 7. С. $787-788$.

44. Розанов Н.Н. Гибридные режимы отражения и пропускания света нелинейными средами // Письма в ЖКТФ. 1977. T. 3. № 12 . С. $583-589$.

45. Розанов Н.Н. Отражение плоской волны нелинейными средами // Письма в ЗЖТФ. 1978. Т. 4. № 2. С. 74-80.

46. Розанов Н.Н. Оптическая бистабильность и гистерезис в распределенных нелинейных системах. М.: Наука, 1997. $336 \mathrm{c.}$

47. Rosanov N.N. Spatial hysteresis and optical patterns. Berlin: Springer, 2002. 308 p.

48. Розанов Н.Н., Ходова Г.В. Нелинейное отражение и пропускание ограниченных пучков света // Опт. спектр. 1979. T. 47. № 3. C. $606-609$.

49. Розанов Н.Н. Диссипативные оптические солитоны. От микро- к нано- и атто-. М.: Физматлит, 2011.536 с.

50. Нелинейности в периодических структурах и метаматериалах / Под ред. Кившаря Ю.С., Розанова Н.Н. М.: Физматлит, 2014. $384 \mathrm{c.}$

51. Розанов Н.Н. Гистерезис профиля температуры при оптическом тепловом пробое полупроводников // Письма в ЗЖТТ. 1980. Т. 6. № 13. С. 778-782.

52.Розанов Н.Н. Гистерезисные явления в распределенных оптических системах // ЗЖЭТФ. 1981. Т. 80 . № 1. C. $96-108$.

53. Fainberg B.D., Rosanov N.N., Veretenov N.A. Light-induced "plasmonic" properties of organic materials: Surface polaritons and switching waves in bistable organic thin films // Appl. Phys. Lett. 2017. V. 110. P. 203301.

54.Розанов Н.Н., Ходова Г.В. Автосолитоны в бистабильных интерферометрах // Опт. спектр. 1988. Т. 65. № 6. C. $1375-1377$.

55. Rosanov N.N., Khodova G.V. Diffractive autosolitons in nonlinear interferometers // JOSA B. 1990. V. 7. № 6. P. 10571065.

56. Rosanov N.N., Fedorov A.V., Khodova G.V. Effects of spatial distributivity in semiconductor optical bistable systems // Phys. Stat. Sol. (b). 1988. V. 150. № 2. P. 545-555.

57. Розанов Н.Н., Ходова Г.В. Волны переключения между стационарными и нестационарными состояниями широкоапертурных бистабильных интерферометров // Квант. электрон. 1989. Т. 16. № 4. С. $785-788$.

58. Розанов Н.Н., Федоров С.В. Дифракционные волны переключения и автосолитоны в лазере с насыщающимся поглощением // Опт. спектр. 1992. Т. 72. № 6. С. 101-106.

59. Lu L., Joannopoulos J.D., Soliačić M. Topological photonics // Nature Photonics. 2014. V. 8. P. 821-829.

60.Faddeev L.D. Quantization of solitons. Princeton preprint IAS-75-QS70. 1975. Princeton: Institute for Advanced Study.

61. Veretenov N.A., Rosanov N.N., Fedorov S.V. Rotating and processing dissipative optical topological 3D solitons // Phys. Rev. Lett. 2016. V. 117. P. 183901. 
62. Veretenov N.A., Fedorov S.V., Rosanov N.N. Topological vortex and knotted dissipative optical 3D solitons generated by 2D vortex solitons // Phys. Rev. Lett. 2017. V. 119. P. 263901.

63. Veretenov N.A., Fedorov S.V., Rosanov N.N. Topological three-dimensional dissipative optical solitons // Phil. Trans. R. Soc. A. 2018. P. 2017.0367.

64. Федоров С.В., Розанов Н.Н., Веретенов Н.А. Структура энергетических потоков в топологических трехмерных диссипативных солитонах // Письма в ЖЭЭТФ. 2018. Т. 107. № 5. С. 342-346.

65. Киселев Ал.С., Киселев Ан.С., Розанов Н.Н. Наноразмерные диссипативные дискретные солитоны в резонансно возбуждаемых Ј-агрегатах // Письма в ЖЖЭТФ. 2008. Т. 87. № 11-12. С. 763-766.

66. Rosanov N.N., Fedorov S.V., Shatsev A.N., Vyssotina N.V. Dissipative molecular solitons // Eur. Phys. J. 2010. V. 59. № 1. P. 3-12.

67. Левинский Б.Н., Нестеров Л.А., Файнберг Б.Д., Розанов Н.Н. Вывод уравнений движения для резонансно возбуждаемых молекулярных Ј-агрегатов при учете многочастичных эффектов // Опт. спектр. 2013. Т. 115 . № 3. C. 464-479.

68. Levinsky B., Fainberg B.D., Nesterov L.A., Rosanov N.N. Two-exciton excited states of J-aggregates in the presence of exciton-exciton annihilation // Chem. Phys. 2016. V. 473. № 7. P. 1-10.

69. Розанов Н.Н. О площади предельно коротких световых импульсов // Опт. спектр. 2009. Т. 107. № 5. С. $761-765$.

70. Rosanov N.N., Kozlov V.V., Wabnitz S. Maxwell-Drude-Bloch dissipative few-cycle optical solitons // Phys. Rev. A. 2010. V. 81. № 4. P. 043815.

71. Розанов Н.Н., Архипов Р.М., Архипов М.В. О законах сохранения в электродинамике сплошных сред // УФН. 2018. T. 188. № 12. DOI: 103367/UFNr2018.07.038386

72. Розанов Н.Н. Взаимодействие интенсивных предельно коротких импульсов с квантовыми объектами // Опт. спектр. 2018. Т. 124. № 1. С. 75-77.

73. Высотина Н.В., Розанов Н.Н., Селенов В.Е. Предельно короткие импульсы усиленной самоиндуцированной прозрачности // Письма в ЖКЭТФ. 2006. Т. 83. № 7-8. С. 337-340.

74. Высотина Н.В., Розанов Н.Н., Селенов В.Е. Предельно короткие диссипативные солитоны в активной среде с квантовыми точками // Опт. спектр. 2009. Т. 106. № 5. С. 793-797.

75. Kozlov V.V., Rosanov N.N., Wabnitz S. Obtaining single-cycle pulses from a mode-locked laser // Phys. Rev. A. 2011. V. 84. № 5. P. 053810.

76. Архипов Р.М., Пахолов А.В., Архипов М.В., Бабушкин И., Толмачев Ю.А., Розанов Н.Н. Генерация униполярных импульсов в нелинейных средах // Письма в ЖЖЭТФ. 2017. Т. 105. № 6. С. 388-400. 